\title{
Impact of breast cancer treatment on fertility
}

Juan de la Haba', Milagros Calderay ${ }^{2}$

${ }^{1}$ Hospital Universitario Reina Sofía, Córdoba, Spain; ${ }^{2}$ Hospital Universitario Ramón y Cajal, Madrid, Spain.

Corresponding author:

Milagros Calderay Domínguez, MD

Department of Gynaecology and Obstetrics

Hospital Universitario Ramón y Cajal

Carretera de Colmenar Viejo KM 9,100

28049-Madrid. Spain

E-mail: $\quad$ mcalderay@gmail.com

Phone: $\quad$ +34913 $\quad$ 36 8105 


\section{Introduction}

Approximately $25 \%$ of breast cancers occur in premenopausal women, and $15 \%$ occur in women younger than 45 years of age [1]. Because the average age of first pregnancy is rising, the percentage of women diagnosed with breast cancer that are of childbearing age or pregnant is also increasing. In addition, the possibility of a woman willing to become pregnant after breast cancer treatment is also rising.

There are no studies showing that later pregnancy has a negative effect on the survival of patients who have been diagnosed and treated for breast cancer. A recent review concluded that there was no adverse effect, and perhaps even a slight protective effect, on survival by childbearing after breast cancer treatment [2]. The superior survival seen in this and other controlled studies may merely reflect a bias towards the selection of healthy patients, but it is also consistent with a potential antitumor effect of pregnancy [2].

The increased survival and decreased mortality of breast cancer patients is partly due to the use of screening programs that allow an earlier diagnosis, and the improvement of treatments, including chemotherapy and hormone therapy [3]. Most cancer therapies have a substantial morbidity on reproductive function, not only because they increase the risk of early menopause, but also because they are associated with a decreased ovarian reserve and a lost of fertility. The impact of infertility on the quality of life of the patient who survives breast cancer explains the increase in the demand for fertility preservation techniques [3].

\section{Chemotherapy-induced amenorrhea (CIA)}

The effects of chemotherapy are reversible in most organs, but not in ovarian tissue. In a significant number of premenopausal women, the administration of adjuvant chemotherapy induces a state of amenorrhea, in many cases irreversible depending on the extent of tissue damage and prior ovarian reserve, which has important short- and 
long-term implications [4] (Table 1). Chemotherapy causes a progressive dose-relate depletion of ovarian follicles and granulosa cells that translates into oligo-amenorrhea and subsequent premature ovarian failure, ultimately leading to what is known as CIA. [5]. From an endocrine point of view, a decrease in estrogen levels with elevated follicule-stimulating hormone $(\mathrm{FSH})$ and luteinizing hormone $(\mathrm{LH})$ levels occurs (i.e. hypergonadotropic hypogonadism).

While there is significant variability in the definition of $\mathrm{CIA}$, some retrospective and prospective studies have reviewed the factors involved in its onset and the degree of reversibility [6-10]. The exact incidence of CIA is difficult to establish because many factors can contribute to it, but the type of chemotherapy, cumulative dose and the patient's age are the main factors that determine the occurrence of CIA. Following chemotherapy, older women have a higher incidence of complete ovarian failure and permanent infertility compared with younger women [3, 11], and it is estimated that the physiological age of the ovaries in a cancer survivor may be 10 years older than the actual chronological age [12].

Therefore, regardless of the beneficial effects that hormonal changes can have as part of the adjuvant endocrine strategy, CIA is an adverse event to take into account for the selection of the best adjuvant treatment.

The abolition of menstruation due to the use of alkylating agents is probably the most reviewed subject in the scientific literature. For patients in whom drugs such as melphalan or cyclophosphamide are used, reported CIA rates range from 25 to $95 \%$ respectively [13]. These rates were lower in younger patients and higher when the number of cycles or dosage was increased [9].

Chemotherapeutic agents can be grouped into three risk groups according to their potential to cause ovarian damage [14]:

1. High Risk: Cyclophosphamide, melphalan, busulfan, chlorambucil, procarbazine and nitrogen mustard are alkylating agents that are associated with an 
increased risk of ovarian failure, with an overall response of 3.98 compared to unexposed women [15].

2. Moderate Risk: Cisplatin causes ovarian failure with an overall response of 1.77. Doxorubicin and paclitaxel also fall into this group.

3. Low risk or no risk: Methotrexate, 5-fluorouracil, bleomycin and actinomycin D, and vinca alkaloids cause ovarian failure with an overall response of about 1.

Regarding specific group data, there are conflicting results regarding the ability of anthracyclines to increase the rate of CIA when these are used in combination with alkylating agents. Thus, in the MA.5 study comparing cyclophosphamide, methotrexate, and fluorouracil (CMF) versus cyclophosphamide, epirubicin, and fluorouracil (CEF), there were higher rates of amenorrhea in women who received anthracyclines (76\% vs $64 \% ; p=0.03)$, although higher doses of alkylating agents were received in the CMF arm [16]. By contrast, in a trial comparing four cycles of doxorubicin and cyclophosphamide (AC) versus six cycles of CMF, the highest percentage of CIA (>12 months) occurred in the CMF arm (34\% vs 69\%) [17]. Age was the most important predictor of CIA in all these studies. For women older than 40 years treated with anthracyclines, CIA rate was 95\%, compared with $30 \%$ in women younger than 40 years.

Other studies have analyzed how the dose and duration of anthracyclines impact on ovarian suppression. The Cancer and Leukemia Group B study (CALGB 8541), which compared three doses of adriamycin $\left(30,40\right.$ and $\left.60 \mathrm{mg} / \mathrm{m}^{2}\right)$, found no differences in rates of CIA [18]. In the French Adjuvant Study Group 01 trial (FASG), three different doses of epirubicin were compared with different numbers of cycles. A relationship between CIA and the cumulative dose of anthracyclines was found [19]. Again, the results are contradictory when the relationship with dose density is studied, especially when maintaining the same cumulative dose of anthracyclines. 
Taxanes are another group of drugs that have been analyzed for this toxicity. For sequential schedules, CIA rates are very similar in patients older than 40 years (85\%). However, there are differences in younger women. A study that compared the same number of cycles of fluorouracil, doxorubicin and cyclophosphamide (FAC) with docetaxel, doxorubicin and cyclophosphamide (TAC) showed that the percentage of amenorrheic patients was higher in the arm that received docetaxel (62\% vs $52 \%$; $p=0.007$ ) [20]. Table 2 shows a summary of studies which have evaluated the incidence of CIA according to different chemotherapy schedules.

The relationship between tamoxifen and amenorrhea is well established, although tamoxifen monotherapy carries a low risk of CIA, which largely depends on the patient's age [21]. The possible mechanism could be an increase in serum estrogen levels and a blockade of the hypothalamic-ovarian axis.

Studies to identify women with diminished ovarian reserve could include measurement of follicle-stimulating hormone, inhibin B or anti-Müllerian hormone levels, or a transvaginal ultrasound to determine the number of antral follicles. Serum antiMüllerian hormone may be a promising marker of ovarian reserve in women after breast cancer treatment because its level decreases with age and in younger patients who survive cancer; other possibilities include measurement of inhibin B levels and antral follicle count [12].

\section{Strategies to preserve fertility}

Young women diagnosed with breast cancer need to be advised by a multidisciplinary team on the impact of treatment on subsequent fertility before starting any treatment, and should be provided with access to specialized units that help them to preserve their fertility.

The choice of the method of preserving fertility depends on the patient's age, her desire to become pregnant, the type of chemotherapeutic treatment to be administered, the 
time available before chemotherapy commences, the amount of time pregnancy must be delayed after treatment, and the potential for the cancer to metastasize to the ovary $[3,22]$.

\section{Embryo and oocyte cryopreservation}

Embryo cryopreservation is currently the only clinically tested procedure. Nevertheless, this approach has several limitations because a partner or a sperm donor is required, and ovarian stimulation is necessary, which may delay the start of chemotherapy by 26 weeks, a situation which may not always be advisable. Overriding all these considerations, however, are the ethical and legal problems associated with embryo cryopreservation $[3,23]$.

Oocyte cryopreservation is an emerging option for preserving fertility in women without partners who do not wish to use donor semen. This procedure also requires ovarian stimulation. The rapid freezing technique of vitrification has presented a breakthrough in oocyte cryopreservation. This technique is fast, safe, effective and less costly. Given the high survival rates, together with the high fertilization rates and better results in terms of healthy births, vitrification is the method of choice for oocyte cryopreservation [24]. The advantages of oocyte cryopreservation are that it requires minimal invasion, oocytes can be preserved for an unlimited time and there is no need for a sperm sample. On the other hand, this method does not maintain hormonal function and is applicable only during childbearing years.

Both embryo and oocyte cryopreservation require ovarian stimulation, but in the case of hormone-dependent breast cancer, the increased estrogen levels produced by ovarian stimulants could contribute to the proliferation of tumor cells and hence should be avoided. To minimize exposure to estrogens, two strategies have been suggested: an unstimulated/natural cycle, and the use of chemoprotective agents with low-dose protocol of in vitro fertilization. The efficacy of natural cycle techniques is hampered by 
high rates of a premature rise in $\mathrm{LH}$ levels and premature ovulation [25]. Tamoxifen and aromatase inhibitors appear to be the best candidates for ovarian stimulation because of both their effectiveness in preventing breast cancer recurrence and their ability to induce ovulation $[3,26]$.

\section{Ovarian cryopreservation}

This technique allows the storage of a large number of primordial follicles for long periods of time before subsequent reimplantation of the preserved ovarian material. It has potential advantages over embyro and oocyte cryopreservation as it can be carried out at any time, does not require ovarian stimulation and does not delay the start of chemotherapy. It is indicated in patients with breast cancer in whom treatment must be started immediately and who therefore cannot undergo ovarian stimulation, as well as in girls and in women for whom sperm donation is unacceptable [27].

Compared to whole ovary cryopreservation, ovarian cortical slice cryopreservation is the preferred technique. It is advisable to analyze samples to confirm the presence of follicles and the absence of metastases. There are several strategies to bring about maturation of oocytes after treatment for cancer such as autotransplantation (orthotopic or heterotopic), xenotransplantation and maturation in vitro [28]. To date, five studies of children born after reimplantation of cryopreserved ovarian tissue have been reported. The number of reimplantations performed by all groups was relatively low due to the amount of time between implantation and the age at which the dissection procedure originally took place [29].

The biggest problem of this technique is that revascularization of the transplanted tissue occurs after several days with consequent ischemia that may induce partial fibrosis and loss of primordial follicles. Another problem that should be considered with autotransplantation of ovarian tissue in women with cancer is the possibility of 
transmitting microscopic metastatic disease. Advantages of this technique are the recovery of hormonal function and the possibility of pregnancy.

Use of gonadotropin-releasing hormone- analogues ( $\mathrm{GnRH}-\mathrm{a}$ )

During treatment with chemotherapy, the use of $\mathrm{GnRH}-\mathrm{a}$ as the only method to preserve fertility is highly controversial. There are several proposed mechanisms of action to explain the protective action of $\mathrm{GnRH}-\mathrm{a}$, including suppression of $\mathrm{FSH}$ secretion and decrease in uterine and ovarian blood flow, with a subsequent decrease in the number of primordial follicles exposed to chemotherapy [30].

There are a limited number of studies supporting the use of $\mathrm{GnRH}$-a to prevent ovarian failure in humans, which have been criticized for the lack of randomization, control groups and follow-up periods [31]. Moreover, GhRH-a causes a prepubertal hormonal situation in adult women, but prepubescent girls who receive high doses of chemotherapy continue to suffer from ovarian failure. Another reason not to recommend the use of $\mathrm{GhRH}-\mathrm{a}$ is because it causes tumor cells to stop in $\mathrm{G} 0$ phase of the cell cycle, making them less sensitive to chemotherapy [32]. Definitive evidence of the role of $\mathrm{GhRH}-\mathrm{a}$ in preserving ovarian function is under evaluation in phase III trials $[31,33]$. We believe that $\mathrm{GnRH}-\mathrm{a}$ should not be offered as the sole method of fertility preservation until the efficacy in protecting ovarian function is fully demonstrated [22].

\section{Conclusions}

Most cancer therapies used have substantial morbidity on later reproductive capacity, not only increasing the risk of early menopause but also decreasing ovarian reserve and loss of fertility. CIA is an important adverse event to take into account in young women when selecting the best adjuvant endocrine strategy. According to the Ethics Committee of the American Society for Reproductive Medicine, physicians should 
inform cancer patients about their reproductive future and options to preserve fertility prior to treatment.

The only clinically established method for fertility preservation is embryo cryopreservation. Experimental procedures such as oocyte and ovarian tissue cryopreservation and $\mathrm{GnRH}$-a should only be offered as part of a clinical trial protocol.

$\mathrm{CIA}$ is a highly complex adverse event that requires close attention, especially with the advent of new chemotherapeutic agents. 


\section{Acknowledgements}

The authors acknowledge the support of Pfizer Spain, which facilitated the necessary meetings to evaluate and discuss all the data presented in this review, and Dr. Fernando Sánchez Barbero from HealthCo SL (Madrid, Spain) for assistance in the preparation of this manuscript. 


\section{References}

1. Hankey BF, Miller B, Curtis R, Kosary C (1994) Trends in breast cancer in younger women in contrast to older women. J Natl Cancer Inst Monogr (16):714

2. Gelber S, Coates AS, Goldhirsch A, Castiglione-Gertsch M, Marini G, Lindtner $J$, et al. (2001) Effect of pregnancy on overall survival after the diagnosis of early-stage breast cancer. J Clin Oncol 19:(6):1671-5

3. Sonmezer M, Oktay K (2006) Fertility preservation in young women undergoing breast cancer therapy. Oncologist 11:(5):422-34

4. Ganz PA, Greendale GA (2001) Menopause and breast cancer: addressing the secondary health effects of adjuvant chemotherapy. J Clin Oncol 19:(14):33035

5. Warne GL, Fairley KF, Hobbs JB, Martin FI (1973) Cyclophosphamide-induced ovarian failure. N Engl J Med 289:(22):1159-62

6. Poikonen P, Saarto T, Elomaa I, Joensuu H, Blomqvist C (2000) Prognostic effect of amenorrhoea and elevated serum gonadotropin levels induced by adjuvant chemotherapy in premenopausal node-positive breast cancer patients. Eur J Cancer 36:(1):43-8

7. Tormey DC, Gray R, Abeloff MD, Roseman DL, Gilchrist KW, Barylak EJ, et al. (1992) Adjuvant therapy with a doxorubicin regimen and long-term tamoxifen in premenopausal breast cancer patients: an Eastern Cooperative Oncology Group trial. J Clin Oncol 10:(12):1848-56

8. Goldhirsch A, Gelber RD, Castiglione M (1990) The magnitude of endocrine effects of adjuvant chemotherapy for premenopausal breast cancer patients. The International Breast Cancer Study Group. Ann Oncol 1:(3):183-8

9. Pagani O, O'Neill A, Castiglione M, Gelber RD, Goldhirsch A, Rudenstam CM, et al. (1998) Prognostic impact of amenorrhoea after adjuvant chemotherapy in 
premenopausal breast cancer patients with axillary node involvement: results of the International Breast Cancer Study Group (IBCSG) Trial VI. Eur J Cancer 34:(5):632-40

10. Richards MA, O'Reilly SM, Howell A, George WD, Fentiman IS, Chaudary MA, et al. (1990) Adjuvant cyclophosphamide, methotrexate, and fluorouracil in patients with axillary node-positive breast cancer: an update of the Guy's/Manchester trial. J Clin Oncol 8:(12):2032-9

11. Marhhom E, Cohen I (2007) Fertility preservation options for women with malignancies. Obstet Gynecol Surv 62:(1):58-72

12. Del Mastro L, Catzeddu T, Venturini M (2006) Infertility and pregnancy after breast cancer: current knowledge and future perspectives. Cancer Treat Rev $32:(6): 417-22$

13. Brincker H, Rose C, Rank F, Mouridsen HT, Jakobsen A, Dombernowsky P, et al. (1987) Evidence of a castration-mediated effect of adjuvant cytotoxic chemotherapy in premenopausal breast cancer. J Clin Oncol 5:(11):1771-8

14. Oktay K, Sonmezer M (2007) Fertility preservation in gynecologic cancers. Curr Opin Oncol 19:(5):506-11

15. Demeestere I, Simon P, Englert Y, Delbaere A (2003) Preliminary experience of ovarian tissue cryopreservation procedure: alternatives, perspectives and feasibility. Reprod Biomed Online 7:(5):572-9

16. Parulekar WR, Day AG, Ottaway JA, Shepherd LE, Trudeau ME, Bramwell V, et al. (2005) Incidence and prognostic impact of amenorrhea during adjuvant therapy in high-risk premenopausal breast cancer: analysis of a National Cancer Institute of Canada Clinical Trials Group Study--NCIC CTG MA.5. J Clin Oncol 23:(25):6002-8 
17. Goodwin PJ, Ennis M, Pritchard KI, Trudeau M, Hood N (1999) Risk of menopause during the first year after breast cancer diagnosis. J Clin Oncol $17:(8): 2365-70$

18. Budman DR, Berry DA, Cirrincione CT, Henderson IC, Wood WC, Weiss RB, et al. (1998) Dose and dose intensity as determinants of outcome in the adjuvant treatment of breast cancer. The Cancer and Leukemia Group B. J Natl Cancer Inst 90:(16):1205-11

19. Fumoleau $\mathrm{P}$, Kerbrat $\mathrm{P}$, Romestaing $\mathrm{P}$, Fargeot $\mathrm{P}$, Bremond $\mathrm{A}$, Namer $\mathrm{M}$, et al. (2003) Randomized trial comparing six versus three cycles of epirubicin-based adjuvant chemotherapy in premenopausal, node-positive breast cancer patients: 10-year follow-up results of the French Adjuvant Study Group 01 trial. J Clin Oncol 21:(2):298-305

20. Martin M, Pienkowski T, Mackey J, Pawlicki M, Guastalla JP, Weaver C, et al. (2005) Adjuvant docetaxel for node-positive breast cancer. N Engl J Med 352:(22):2302-13

21. Swain SM, Land SR, Ritter MW, Costantino JP, Cecchini RS, Mamounas EP, et al. (2009) Amenorrhea in premenopausal women on the doxorubicin-andcyclophosphamide-followed-by-docetaxel arm of NSABP B-30 trial. Breast Cancer Res Treat 113:(2):315-20

22. Lee SJ, Schover LR, Partridge AH, Patrizio P, Wallace WH, Hagerty K, et al. (2006) American Society of Clinical Oncology recommendations on fertility preservation in cancer patients. J Clin Oncol 24:(18):2917-31

23. Bankowski BJ, Lyerly AD, Faden RR, Wallach EE (2005) The social implications of embryo cryopreservation. Fertil Steril 84:(4):823-32

24. Kim SS (2006) Fertility preservation in female cancer patients: current developments and future directions. Fertil Steril 85:(1):1-11 
25. Pelinck MJ, Hoek A, Simons AH, Heineman MJ (2002) Efficacy of natural cycle IVF: a review of the literature. Hum Reprod Update 8:(2):129-39

26. Casper RF (2007) Aromatase inhibitors in ovarian stimulation. J Steroid Biochem Mol Biol 106:(1-5):71-5

27. Donnez J, Jadoul P, Squifflet J, Van Langendonckt A, Donnez O, Van Eyck AS, et al. (2009) Ovarian tissue cryopreservation and transplantation in cancer patients. Best Pract Res Clin Obstet Gynaecol

28. Kim SS, Battaglia DE, Soules MR (2001) The future of human ovarian cryopreservation and transplantation: fertility and beyond. Fertil Steril 75:(6):1049-56

29. Novella-Maestre E, Sánchez M. Estadop actual de la congelación de la corteza ovárica. In: García Velasco JA, editor. Cuadernos de Medicina Reproductiva; 2009. p. 83-90.

30. Tocino A, Sánchez M, Caligara Z. Uso de los análogos de la GnRH en la preservación de la fertilidad. In: García Velasco JA, editor. Cuadernos de Medicina Reproductiva; 2009. p. 61-69.

31. Gerber B, Dieterich M, Muller H, Reimer T (2008) Controversies in preservation of ovary function and fertility in patients with breast cancer. Breast Cancer Res Treat 108:(1):1-7

32. So WK, Cheng JC, Poon SL, Leung PC (2008) Gonadotropin-releasing hormone and ovarian cancer: a functional and mechanistic overview. Febs $\mathrm{J}$ 275:(22):5496-511

33. Manger K, Wildt L, Kalden JR, Manger B (2006) Prevention of gonadal toxicity and preservation of gonadal function and fertility in young women with systemic lupus erythematosus treated by cyclophosphamide: the PREGO-Study. Autoimmun Rev 5:(4):269-72 
34. Castiglione-Gertsch M, O'Neill A, Price KN, Goldhirsch A, Coates AS, Colleoni M, et al. (2003) Adjuvant chemotherapy followed by goserelin versus either modality alone for premenopausal lymph node-negative breast cancer: a randomized trial. J Natl Cancer Inst 95:(24):1833-46

35. Bonadonna G, Moliterni A, Zambetti M, Daidone MG, Pilotti S, Gianni L, et al. (2005) 30 years' follow up of randomised studies of adjuvant CMF in operable breast cancer: cohort study. BMJ 330:(7485):217

36. Roche $\mathrm{H}$, Kerbrat $\mathrm{P}$, Bonneterre J, Fargeot $\mathrm{P}$, Fumoleau $\mathrm{P}$, Monnier $\mathrm{A}$, et al. (2006) Complete hormonal blockade versus epirubicin-based chemotherapy in premenopausal, one to three node-positive, and hormone-receptor positive, early breast cancer patients: 7-year follow-up results of French Adjuvant Study Group 06 randomised trial. Ann Oncol 17:(8):1221-7

37. Berliere M, Dalenc F, Malingret N, Vindevogel A, Piette P, Roche H, et al. (2008) Incidence of reversible amenorrhea in women with breast cancer undergoing adjuvant anthracycline-based chemotherapy with or without docetaxel. BMC Cancer 8:56

38. Tham YL, Sexton K, Weiss H, Elledge R, Friedman LC, Kramer R (2007) The rates of chemotherapy-induced amenorrhea in patients treated with adjuvant doxorubicin and cyclophosphamide followed by a taxane. Am J Clin Oncol $30:(2): 126-32$

39. Perez-Fidalgo JA, Rosello S, Garcia-Garre E, Jorda E, Martin-Martorell P, Bermejo B, et al. (2009) Incidence of chemotherapy-induced amenorrhea in hormone-sensitive breast cancer patients: the impact of addition of taxanes to anthracycline-based regimens. Breast Cancer Res Treat 
Table 1. Side effects of amenorrhea.

Side effects of amenorrhea

Osteoporosis

Arthralgia

Increased cardiovascular morbidity

Hot Flushes

Genitourinary dysfunctions

Sexual dysfunctions

Sterility

Insomnia

Anxiety

Depression 
Table 2. Incidence of chemotherapy-induced amenorrhea according to different chemotherapy schedules.

\begin{tabular}{|c|c|c|c|c|}
\hline Author & Treatment regimen & № Cycles & Age & $\%$ of Amenorrhea \\
\hline \multirow[b]{2}{*}{ Castiglione [34] } & \multirow[b]{2}{*}{ CMF } & \multirow[b]{2}{*}{6} & $>40$ & 90 \\
\hline & & & $\leq 40$ & 50 \\
\hline \multirow[b]{2}{*}{ Bonadonna [35] } & \multirow[b]{2}{*}{ CMF } & \multirow[b]{2}{*}{12} & $>40$ & 96 \\
\hline & & & $\leq 40$ & 40 \\
\hline \multirow{2}{*}{ Roche [36] } & \multirow{2}{*}{ CEF } & \multirow{2}{*}{6} & & 76 \\
\hline & & & $\leq 40$ & 38 \\
\hline \multirow{5}{*}{ Berliere [37] } & \multirow{2}{*}{ CEF100 $\times 6$} & \multirow{3}{*}{6} & $>40$ & 72 \\
\hline & & & $\leq 40$ & 10 \\
\hline & vs & & & \\
\hline & \multirow{2}{*}{$\mathrm{CEF} 100 \times 3 \rightarrow \mathrm{T} 100 \times 3$} & \multirow{2}{*}{3} & $>40$ & 71 \\
\hline & & & $\leq 40$ & 20 \\
\hline \multirow{2}{*}{ Martin [20] } & FAC vs & 6 & & 52 \\
\hline & TAC & 6 & & 62 \\
\hline \multirow{5}{*}{ Tham [38] } & \multirow{2}{*}{$A C$} & \multirow[b]{2}{*}{4} & $>40$ & 81 \\
\hline & & & $<10$ & $\Delta 4$ \\
\hline & vs & \multirow[b]{3}{*}{8} & & 44 \\
\hline & \multirow{2}{*}{$A C \rightarrow(D$ or $P)$} & & $>40$ & 85 \\
\hline & & & $\leq 40$ & 61 \\
\hline \multirow{3}{*}{ Perez-Fidalgo [39] } & Anthracyclines & & & 76 \\
\hline & vs & & & \\
\hline & Anthracyclines + Taxanes & & & 83 \\
\hline
\end{tabular}

